\title{
TOLERÂNCIA DE GENÓTIPOS DE PIMENTÃO AO BAIXO TEOR DE FÓSFORO NO SOLO ${ }^{(1)}$
}

\author{
VALTER RODRIGUES OLIVEIRA ${ }^{(2)}$, VICENTE WAGNER DIAS CASALI ${ }^{(3)}$, PAULO ROBERTO \\ GOMES PEREIRA $^{(3)}$, COSME DAMIÃO CRUZ ${ }^{(4)} \&$ NÁDJA DE MOURA PIRES ${ }^{(5)}$
}

\section{RESUMO}

Vinte e dois genótipos de pimentão (Capsicum annuum L.) foram cultivados em vasos com 2,45 $\mathrm{dm}^{3}$ de solo em seis doses de fósforo (P) $\left(25,50,100,200,300\right.$ e $\left.600 \mathrm{mg} \cdot \mathrm{dm}^{-3} \mathrm{P}\right)$. Avaliou-se o desempenho dos genótipos em cada dose de $\mathrm{P}$ aplicada pelas características que expressam o crescimento e a eficiência nutricional. Houve acentuada diferença entre os genótipos nas várias doses de $\mathrm{P}$, tanto na acumulação de matéria seca total quanto nas demais características relacionadas com o crescimento, enquanto, para as características nutricionais, houve menores diferenças. Entre as diferentes doses, a de $200 \mathrm{mg} \cdot \mathrm{dm}^{-3} \mathrm{P}$, pela maior precisão experimental e maior variabilidade genotípica, foi escolhida como a mais indicada para a identificação de genótipos tolerantes à baixa disponibilidade de $\mathrm{P}$ e para estudos genéticos. Pela comparação das médias de matéria seca total e com base em funções discriminantes (técnica de análise multivariada proposta por T.W. Anderson), identificaram-se seis genótipos tolerantes, dez moderadamente tolerantes e seis intolerantes ao baixo teor de P. Aparentemente, um único fator foi o responsável pela diferença no crescimento entre os genótipos tolerantes e intolerantes. Os tolerantes, que foram 2,34 vezes mais eficientes na produção de matéria seca total, acumularam 2,38 vezes mais $P$, em média, do que os intolerantes, provavelmente em razão da sua maior biomassa de raiz. Diferenças no uso interno do $P$ não contribuíram para a diferenciação dos genótipos tolerantes e intolerantes.

Termos de indexação: pimentão, Capsicum annuum L., tolerância, fósforo, deficiência de fósforo.

\footnotetext{
(1) Recebido para publicação em 25 de junho de 1997 e aceito em 22 de março de 1999.

${ }^{(2)}$ Centro Tecnológico do Centro-Oeste, Empresa de Pesquisa Agropecuária de Minas Gerais, Caixa Postal 295, 35701-970 Sete Lagoas (MG).

(3) Departamento de Fitotecnia, Universidade Federal de Viçosa, Avenida Peter Henry Rolfs, s/n, 36571-000 Viçosa (MG).

(4) Departamento de Biologia Geral, Universidade Federal de Viçosa.

(5) Centro Nacional de Pesquisa de Milho e Sorgo, Empresa Brasileira de Pesquisa Agropecuária, Caixa Postal 151, 35701-970 Sete Lagoas (MG).
} 


\title{
ABSTRACT \\ GENOTYPE TOLERANCE OF SWEET PEPPER TO LOW PHOSPHORUS CONTENT IN SOIL
}

\begin{abstract}
Twenty two genotypes of sweet pepper (Capsicum annuиm L.) were grown in pots containing $2.45 \mathrm{dm}^{3}$ soil, in which six phosphorus (P) levels were applied $(25,50,100,200$, 300 and $600 \mathrm{mg} \cdot \mathrm{dm}^{-3} \mathrm{P}$ ). For each P level, the genotype performance was evaluated according to the characteristics expressing growth and nutritional efficiency. For several P levels, there was an occurrence of great differences among genotypes either in total dry matter accumulation and other characteristics related to growth, whereas low differences were observed in the nutritional characteristics. Among all $\mathrm{P}$ levels applied, $200 \mathrm{mg} \cdot \mathrm{dm}^{-3} \mathrm{P}$ was the most adequate for both identification of genotypes which are tolerant to low $\mathrm{P}$ availability and genetic studies, for its experimental accuracy and greater genotypic variability provided. By comparing the averages of total dry matter and based on discriminatory functions (multivariate analysis technique proposed by T. W. Anderson), six genotypes were identified as tolerant, ten as moderately tolerant and six as intolerant to low P contents. Apparently, only one factor was responsible for the difference in growing, which was observed between tolerant and intolerant genotypes. The tolerant genotypes which showed to be 2.34 times more efficient in producing total dry matter, also accumulated 2.38 times more $\mathrm{P}$ in the average, than those intolerant, probably due to greater biomass of tolerant-genotype roots. Differences in internal P use did not contribute for the differences between tolerant and intolerant genotypes.
\end{abstract}

Index terms: sweet pepper, Capsicum annuиm L., tolerance, phosphorus, phosphorus deficiency.

\section{INTRODUÇÃO}

Uma grande porção dos solos das regiões tropicais e subtropicais é caracterizada pela baixa disponibilidade de fósforo $(\mathrm{P})$, cuja correção normalmente se faz pela adição de altas doses de fertilizantes fosfatados, uma solução econômica e ambientalmente, de modo geral, insatisfatória. Alternativas têm sido constantemente sugeridas, entre elas o desenvolvimento de cultivares mais eficientes (Silva \& Gabelman, 1992; Fageria \& Baligar, 1997). Cultivares de pimentão tolerantes ao baixo teor de $\mathrm{P}$ devem ser de considerável interesse para melhoristas, produtores e consumidores, por oferecer economia de nutrientes, com conseqüente redução no custo de produção e preservação dos recursos naturais.
No aspecto nutricional, um genótipo tolerante à baixa disponibilidade de $\mathrm{P}$ no solo pode ser definido como aquele capaz de crescer satisfatoriamente, sendo eficiente em absorver o $\mathrm{P}$, distribuí-lo internamente e/ou fazer melhor uso do $\mathrm{P}$ absorvido para produzir biomassa. A ocorrência de variabilidade genotípica quanto à tolerância ao baixo teor de $\mathrm{P}$, fundamental para o estabelecimento de programas de melhoramento objetivando o desenvolvimento de cultivares tolerantes, tem sido relatada em feijão (Whiteaker et al., 1976), tomate (Coltman et al., 1985), alface (Buso \& Bliss, 1988), milho (Silva \& Gabelman, 1992), arroz (Chaubey et al., 1994) e pimentão (Moura, 1996).

$\mathrm{Na}$ avaliação da diversidade genotípica quanto ao crescimento sob limitada disponibilidade de P, têm-se utilizado, preferencialmente, condições controladas 
de cultivo, como cultura hidropônica (Furlani et al., 1985; Gabelman et al., 1986) e cultivo no sistema areia-alumina (Buso \& Bliss, 1988; Silva \& Gabelman, 1992) e, mais raramente, no solo, em vasos (Föhse et al., 1988) e no solo, em campo (Oliveira et al., 1987; Schettini et al., 1987 ). A preferência por sistemas de cultivo mais artificiais tem sido justificada pela necessidade de redução dos efeitos ambientais, em função da complexidade da relação solo-raiz (Whiteaker et al., 1976). Embora o solo, pelos problemas de heterogeneidade, complexidade, reprodutividade dos resultados e recuperação do sistema radicular, não seja o preferido para a avaliação da tolerância, certamente é o mais indicado, quando o objetivo é a identificação de progenitores tolerantes à baixa disponibilidade de $\mathrm{P}$ para programas de melhoramento.

O objetivo deste trabalho foi determinar tanto a dose de $\mathrm{P}$ aplicada no solo, em vaso, mais adequada para identificar, por critérios univariado e multivariado, genótipos de pimentão tolerantes e intolerantes à baixa disponibilidade de $\mathrm{P}$, como o mecanismo responsável pela tolerância.

\section{MATERIAL E MÉTODOS}

O ensaio foi realizado em casa de vegetação, no município de Viçosa (MG), de 21 de fevereiro a 9 de maio de 1995. Vinte e dois genótipos de pimentão (Capsicum annuum L.), pertencentes à coleção de Capsicum do Banco de Germoplasma de Hortaliças da Universidade Federal de Viçosa, foram avaliados em seis diferentes doses de $\mathrm{P}$, aplicadas em vasos com $2,45 \mathrm{dm}^{3}$ de solo. Os genótipos identificados com o prefixo P-141 e P-142 são linhagens originadas de cruzamentos múltiplos envolvendo os genitores BGH 18, 'Avelar', 'Pimiento Seleção Stain', 'Yolo Wonder', 'Aconcágua', 'Chineese Giant', 'Pimiento', 'Agronômico 10G' e 'São Carlos'. O acesso BGH 4531 originou-se da seleção em 'Rubyking'. Todos os genótipos possuem frutos cônicos, de coloração verde-escura uniforme e brilhante, com reduzida presença de deformações e produtividade variando de média a alta (Oliveira, 1997).

O solo usado foi um latossolo variação Una (LU), coletado no município de Viçosa, à profundidade de $20-50 \mathrm{~cm}$, com as seguintes características químicas antes da aplicação dos tratamentos com $\mathrm{P}$ : $\mathrm{pH}\left(\mathrm{H}_{2} \mathrm{O}\right)(1: 2,5)=5,1 ; \mathrm{P}=2,0 \mathrm{mg} \cdot \mathrm{dm}^{-3} ; \mathrm{K}^{+}=40,0$ $\mathrm{mg} \cdot \mathrm{dm}^{-3} ; \mathrm{Ca}^{2+}=17,0 \mathrm{mmol} \cdot \mathrm{dm}^{-3} ; \mathrm{Mg}^{2+}=6,0$ $\mathrm{mmol}_{\mathrm{c}} \cdot \mathrm{dm}^{-3} ; \mathrm{Al}^{3+}=1,0 \mathrm{mmol}_{\mathrm{c}} \cdot \mathrm{dm}^{-3} ; \mathrm{H}^{+}+\mathrm{Al}^{3+}=40,4$ $\mathrm{mmol}_{\mathrm{c}} \cdot \mathrm{dm}^{-3}$. Na análise, empregaram-se os seguintes extratores: P e K ${ }^{+}$, Mehlich-1; $\mathrm{Al}^{3+}, \mathrm{Ca}^{2+} \mathrm{e} \mathrm{Mg}^{2+}$, solução de $\mathrm{KCl} 1,0$ mol. $\mathrm{L}^{-1} ; \mathrm{H}^{+}+\mathrm{Al}^{3+}$, solução de oxalato de cálcio 0,5 mol.L $\mathrm{L}^{-1}, \mathrm{pH} 7,0$.

Após seco ao ar, o solo foi destorroado, passado em peneira com malhas de $4 \mathrm{~mm}$ e homogeneizado, recebendo o $\mathrm{P}$ na forma de superfosfato triplo moído,

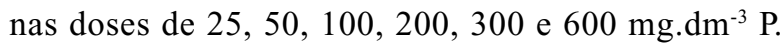
Essas doses foram escolhidas na expectativa de abranger desde a deficiência até uma concentração adequada do elemento. O cálcio foi mantido constante, usando-se o $\mathrm{CaCl}_{2} \cdot 2 \mathrm{H}_{2} \mathrm{O}$, mantendo-se uma relação $\mathrm{Ca}: \mathrm{Mg}$ de 2:1 em equivalente. Como fonte de $\mathrm{Mg}$, utilizaram-se o $\mathrm{MgSO}_{4} \cdot 7 \mathrm{H}_{2} \mathrm{O}$ e o $\mathrm{MgCl}_{2} \cdot 6 \mathrm{H}_{2} \mathrm{O}$. Os demais macro e micronutrientes fornecidos foram: 100,0 mg.dm ${ }^{-3} \mathrm{~N}\left(\mathrm{NH}_{4} \mathrm{NO}_{3}\right), 160,0 \mathrm{mg} \cdot \mathrm{dm}^{-3} \mathrm{~K}\left(\mathrm{~K}_{2} \mathrm{SO}_{4}\right), 80,0$ $\mathrm{mg} . \mathrm{dm}^{-3} \mathrm{~S}\left(\mathrm{~K}_{2} \mathrm{SO}_{4}, \mathrm{MgSO}_{4} \cdot 7 \mathrm{H}_{2} \mathrm{O}, \mathrm{ZnSO}_{4} \cdot 7 \mathrm{H}_{2} \mathrm{O}\right.$, $\left.\mathrm{CuSO}_{4}\right), 0,81 \mathrm{mg} \cdot \mathrm{dm}^{-3} \mathrm{~B}\left(\mathrm{H}_{3} \mathrm{BO}_{3}\right), 1,33 \mathrm{mg} \cdot \mathrm{dm}^{-3} \mathrm{Cu}$ $\left(\mathrm{CuSO}_{4}\right), 3,66 \mathrm{mg} \cdot \mathrm{dm}^{-3} \mathrm{Mn}\left(\mathrm{MnCl}_{2} \cdot 4 \mathrm{H}_{2} \mathrm{O}\right), 0,15$ mg.dm $\left.{ }^{-3} \mathrm{Mo}\left[\left(\mathrm{NH}_{4}\right)_{6} \cdot \mathrm{Mo}_{7} \cdot \mathrm{O}_{24} \cdot 4 \mathrm{H}_{2} \mathrm{O}\right)\right]$ e $4,0 \mathrm{mg} \cdot \mathrm{dm}^{-3} \mathrm{Zn}$ $\left(\mathrm{ZnSO}_{4} \cdot 7 \mathrm{H}_{2} \mathrm{O}\right)$. Efetuou-se a semeadura 25 dias após a adubação, utilizando seis sementes por vaso. Quando as plântulas apresentavam duas folhas definitivas, realizou-se o desbaste, deixando uma planta por vaso. O nitrogênio foi fornecido, na sua totalidade, em cobertura, em aplicações a intervalos médios de dez dias, com início aos quinze dias da semeadura. Durante o ensaio, a umidade do solo foi mantida em torno de $85 \%$ da capacidade de campo, controlada por meio de pesagem dos vasos a intervalos de quatro dias. $\mathrm{O}$ delineamento adotado foi o de blocos completos casualizados, com quatro repetições, sendo a parcela experimental composta de um vaso com uma planta, totalizando 528 vasos. 
As plantas, colhidas aos 65 dias após a germinação, foram separadas em folhas, caule e raízes, secas em estufa com ventilação forçada a $70^{\circ} \mathrm{C}$ para determinação da massa seca. As amostras foram moídas e submetidas à análise de $\mathrm{P}$, colorimetricamente, pelo método da redução do fosfomolibdato pela vitamina C, descrito por Braga \& Defelipo (1974). A produção de matéria seca e o conteúdo de $\mathrm{P}$ foram usados para derivar medidas adicionais relacionadas à eficiência de absorção, translocação e emprego de P.

As características avaliadas foram: altura da planta (AP), em cm, medida do nível do solo ao ponto mais alto da planta; diâmetro do caule (DC), em $\mathrm{mm}$, medido próximo ao nível do solo; área foliar (AF), em $\mathrm{cm}^{2} /$ planta; matéria seca das raízes (MSR), em g/planta; matéria seca da parte aérea (MSA), em g/planta, obtida pela soma da matéria seca do caule e das folhas; matéria seca total (MST), em g/planta, pela soma da matéria seca das raízes, do caule e das folhas; razão entre a matéria seca da raiz e da parte aérea (RRPA); conteúdo de P total na planta (CPP), em mg/ planta; coeficiente de absorção de $\mathrm{P}$ (CAP), mediante a razão $\mathrm{mg}$ de $\mathrm{P}$ total/g de matéria seca das raízes; coeficiente de translocação de P (CTP), por meio da razão $\mathrm{mg}$ de $\mathrm{P}$ na parte aérea/mg de $\mathrm{P}$ total; e coeficiente de utilização do $\mathrm{P}$ total para a produção da matéria seca total (CUPT), obtido pela razão g de matéria seca total $/ \mathrm{mg}$ de $\mathrm{P}$ total.

Para a interpretação dos dados, inicialmente, realizou-se a análise da variância de cada uma das características avaliadas, em cada dose de $\mathrm{P}$, adotando-se o modelo estatístico fixo. Adicionalmente, da análise da variância, estimaram-se, para cada característica e dose de $\mathrm{P}$, as seguintes características: (a) componente de variação fenotípico entre as médias dos tratamentos $\left(\hat{\sigma}_{\mathrm{f}}^{2}\right)$, dado pela razão $\mathrm{QMG} / \mathrm{r}$; (b) componente quadrático $\left(\hat{\phi}_{\mathrm{g}}\right)$ que expressa a variabilidade genotípica entre as médias de genótipos, pela razão (QMG-QMR)r; (c) coeficiente de variação experimental $\left(\mathrm{CV}_{\mathrm{e}}\right)$, pela expressão $100 \sqrt{\mathrm{QMR}} / \hat{\mathrm{m}}$; (d) coeficiente de variação genotípico $\left(\mathrm{CV}_{\mathrm{g}}\right)$, pela expressão $100 \sqrt{\hat{\phi}_{\mathrm{g}}} / \hat{\mathrm{m}}$; (e) razão $\mathrm{CV}_{\mathrm{g}} / \mathrm{CV}_{\mathrm{e}}$; e (f) coeficiente de determinação genotípico $\left(\mathrm{H}^{2}\right)$, dado pela razão $\hat{\phi}_{\mathrm{g}} / \hat{\sigma}_{\mathrm{f}^{*}}$.
Nas expressões anteriores, QMG, QMR, r e m correspondem, respectivamente, ao quadrado médio de genótipos, ao quadrado médio do resíduo, ao número de repetições e à média geral da característica avaliada. Esses componentes e coeficientes foram utilizados na identificação da dose de $\mathrm{P}$ mais adequada para a discriminação genotípica e para estudos genéticos quanto à tolerância à baixa disponibilidade de $\mathrm{P}$. Considerou-se a dose mais adequada aquela em que se detectou maior precisão experimental e maior manifestação da variabilidade genotípica.

Determinou-se a classificação dos genótipos quanto à tolerância à baixa disponibilidade de $\mathrm{P}$, na dose escolhida,mediante dois procedimentos: pela comparação das médias de matéria seca total dos genótipos, utilizando-se o teste de Tukey, a 5\% de probabilidade, a técnica multivariada, denominada função discriminante, descrita por Anderson (1958). Pela comparação das médias, genótipos tolerantes foram aqueles com maiores médias de matéria seca total e, intolerantes, os de menores médias. Com a técnica da função discriminante, a finalidade foi classificar genótipos de comportamento desconhecido em grupos conhecidos, fazendo-se uso simultâneo de um conjunto de características tomadas em cada genótipo. Para a aplicação dessa técnica, é necessário o conhecimento prévio dos genótipos que sabidamente pertencem a cada um dos grupos aos quais se pretende alocar os genótipos de comportamento desconhecido. No presente caso, objetivou-se alocar os genótipos avaliados em dois grupos: tolerantes e intolerantes ao baixo teor de $\mathrm{P}$ no solo, tomando-se como padrão de tolerância e intolerância, respectivamente, os genótipos de comportamento consistentemente superior e inferior, neste ensaio e em outros anteriores, em condições de campo e de casa de vegetação (dados não apresentados), a partir dos quais foram estimadas as funções discriminantes $\mathrm{D}_{\mathrm{t}}(\mathrm{x})$ e $\mathrm{D}_{\mathrm{i}}(\mathrm{x})$, utilizadas para a obtenção dos escores para a classificação dos genótipos nos grupos tolerantes e intolerantes respectivamente. Assim, classificou-se determinado genótipo como tolerante ao baixo teor de $\mathrm{P}$, se $\mathrm{D}_{\mathrm{t}}(\mathrm{x})$ foi maior que $\mathrm{D}_{\mathrm{i}}(\mathrm{x})$, e intolerante, em caso contrário. As probabilidades de os genótipos pertencerem aos grupos tole- 
rantes e intolerantes foram consideradas a priori iguais a 0,5 por não se ter informação prévia sobre os genótipos a serem classificados. Todas as análises foram realizadas com o auxílio do programa computacional Genes (Cruz, 1997).

\section{RESULTADOS E DISCUSSÃO}

\subsection{Dose mais adequada de $\mathbf{P}$ aplicada no solo para a discriminação genotípica}

A baixa fertilidade natural do solo em P $(2,0$ $\mathrm{mg} . \mathrm{dm}^{-3} \mathrm{P}$ ), associada à alta capacidade de adsorção do $\mathrm{P}$ em formas não disponíveis para as plantas, impossibilitou a produção de material vegetal suficiente para as análises químicas na dose de $25 \mathrm{mg} \cdot \mathrm{dm}^{-3} \mathrm{P}$, sendo os resultados discutidos apenas para as demais doses.

A significância da estatística F (Quadro 1) evidencia a ocorrência de diferenças entre as médias dos genótipos, nas várias doses, tanto para MST quanto para as demais características de crescimento avaliadas, como AP, DC, AF, MSR, MSA e RRPA, bem como para CPP. Em relação às demais características nutricionais, como CAP, CTP e CUPT, essa variabilidade foi menos evidente, principalmente nas doses de 200 e $300 \mathrm{mg} \cdot \mathrm{dm}^{-3}$ P. Ampla variabilidade na resposta ao P no solo foi também verificada por Moura (1996), em ensaio com outros genótipos de pimentão com a mesma genealogia dos avaliados no presente trabalho.

Os intervalos de variação entre as médias de genótipos, das diversas características, são também apresentados no Quadro 1. Verifica-se que, de modo geral, os maiores intervalos de variação no desempenho dos genótipos foram observados nas características de crescimento AF, MSR, MSA e MST e no conteúdo de $\mathrm{P}$ total na planta.

Considerando que a melhor dose de $\mathrm{P}$ para a discriminação genotípica, quanto à tolerância à baixa disponibilidade de $\mathrm{P}$, é aquela abaixo da ótima para o crescimento, que proporcione boa precisão experimental $\left(<\mathrm{CV}_{\mathrm{e}}\right)$ e que permita a maior expressão da variabilidade genotípica da matéria seca total e das demais características avaliadas, a dose de $200 \mathrm{mg} \cdot \mathrm{dm}^{-3} \mathrm{P}$ mostrou-se a mais adequada. Os elevados valores da estatística $\mathrm{F}$ e dos parâmetros $\mathrm{H}^{2}$ e $\mathrm{CV}_{\mathrm{g}} / \mathrm{CV}_{\mathrm{e}}$, principalmente para MST (Quadro 1), evidencia uma situação bastante favorável à identificação de genótipos contrastantes nessa dose de P no solo. Estimativas elevadas do coeficiente de determinação genotípico $\left(\mathrm{H}^{2}\right)$ indicam que a maior parte da variação entre as médias de genótipos é de natureza genética, enquanto valores superiores à unidade na relação $\mathrm{CV}_{\mathrm{g}} / \mathrm{CV}_{\mathrm{e}}$ mostram que a variação genotípica supera a ambiental, sendo ambas as situações indicativas de possibilidade de sucesso na identificação de genótipos superiores (Vencovsky, 1987).

\subsection{Classificação dos genótipos quanto à tole- rância ao baixo teor de $\mathbf{P}$ no solo}

Comparações da matéria seca total acumulada foram inicialmente utilizadas para classificar os genótipos como tolerantes e intolerantes ao baixo teor de $\mathrm{P}$ no solo. As médias de MST dos genótipos cultivados em solo com suprimento deficiente $\left(200 \mathrm{mg} \cdot \mathrm{dm}^{-3} \mathrm{P}\right)$ e com suprimento adequado de $\mathrm{P}\left(600 \mathrm{mg}^{\mathrm{dm}} \mathrm{dm}^{-3} \mathrm{P}\right)$ estão apresentadas no Quadro 2. Observa-se que, em solo com suprimento adequado, à exceção dos genótipos 11 e 14, que foram os extremos na acumulação de matéria seca total, os demais foram similares, sendo os genótipos separados em três grupos $(\mathrm{a}, \mathrm{b}, \mathrm{c})$, pelo teste de Tukey $(\mathrm{P}<0,05)$. Em solo com suprimento deficiente, houve acentuada diferença nas médias de MST, com o teste de comparação das médias (Tukey, $\mathrm{P}<0,05$ ), separando os genótipos em seis grupos (a, b, c, d, e, f). Esse comportamento favorece a identificação de indivíduos tolerantes ao estresse nutricional, pois mostra que genótipos com capacidade produtiva semelhante sob adequada disponibilidade do nutriente variam amplamente em solo com suprimento deficiente, sendo esta condição desejável para a seleção dos genótipos quanto à tolerância à limitada disponibilidade de P no solo (Buso \& Bliss, 1988; Gourley et al., 1993). 


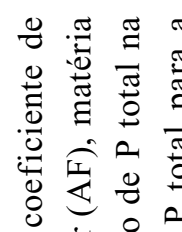

$>$ 章 总 。

บ

$>$ ग

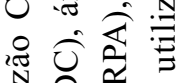

철

نे

元

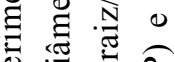

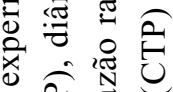

$\circ$ 过

:

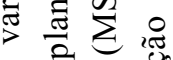

ช 즁

苛营

등

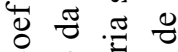

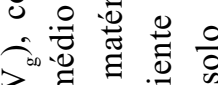

已 0 㐫远

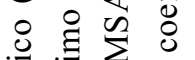

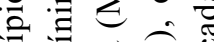

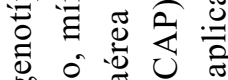

政

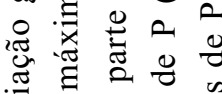

㱐

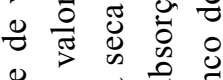

Ð 0.0

ฮี

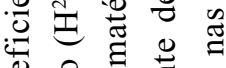

过苛

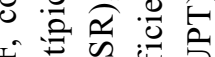

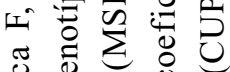

氙

荀

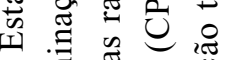

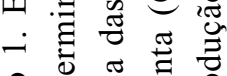

은 离

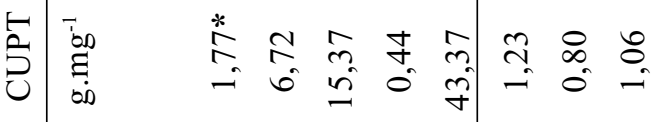

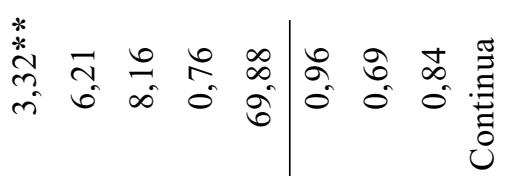

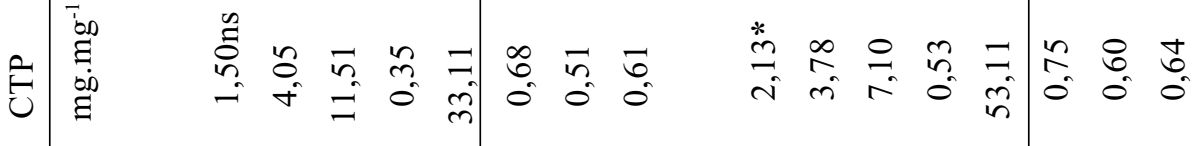

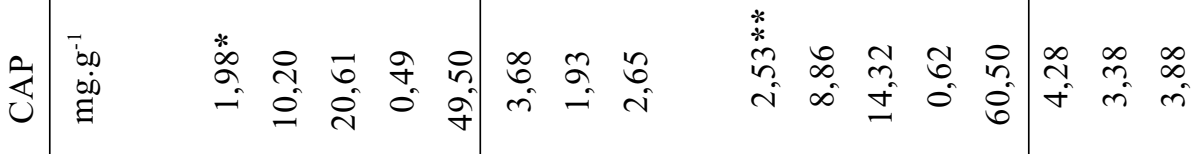

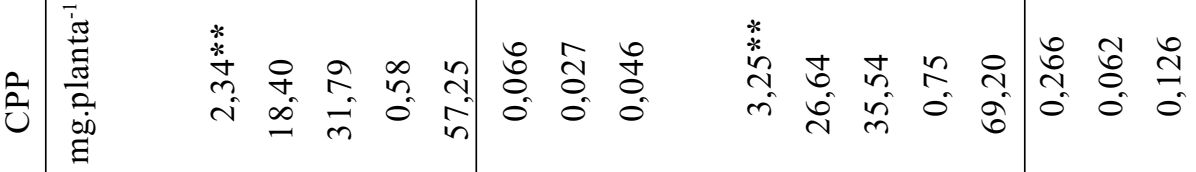

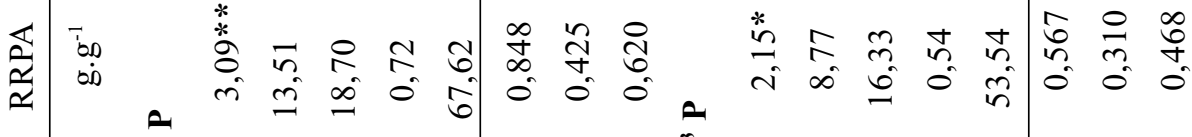

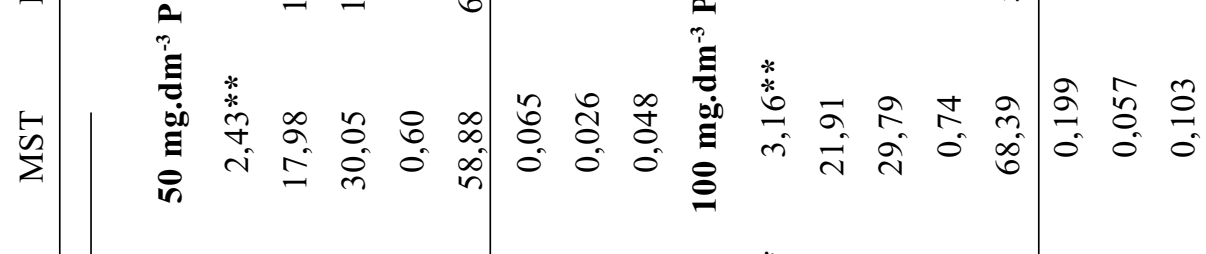

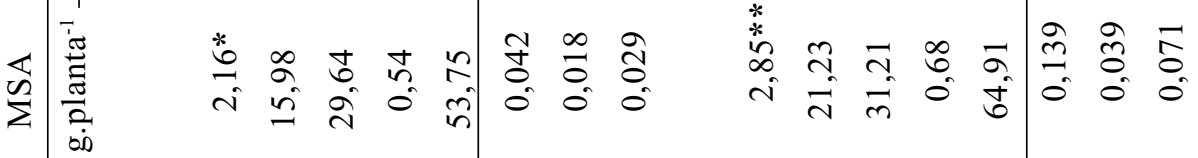

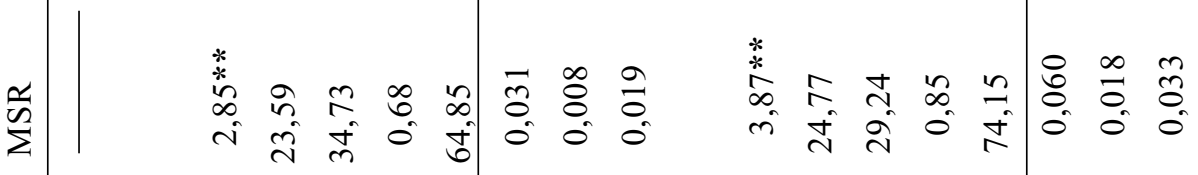

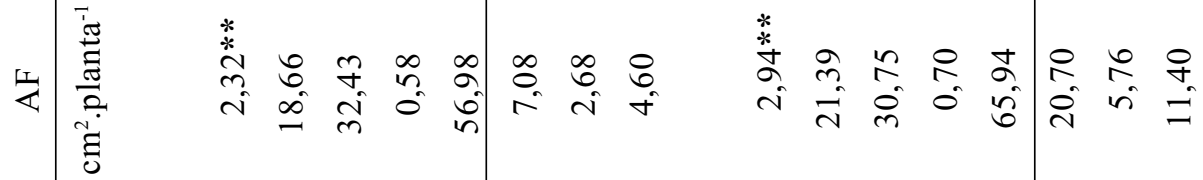

ن

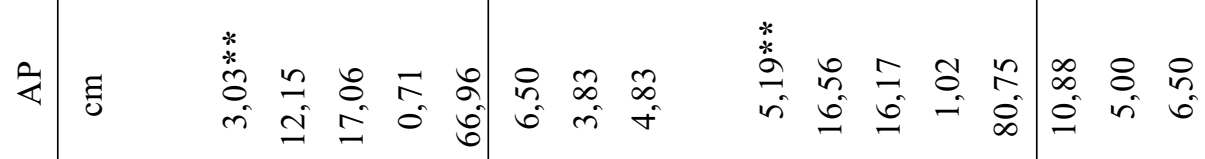

离

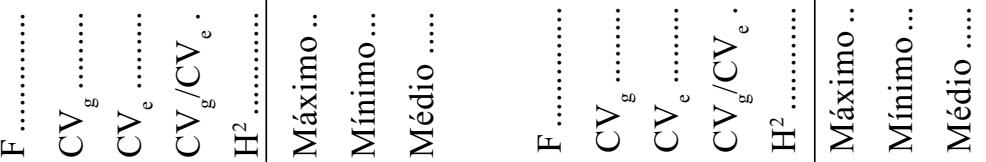



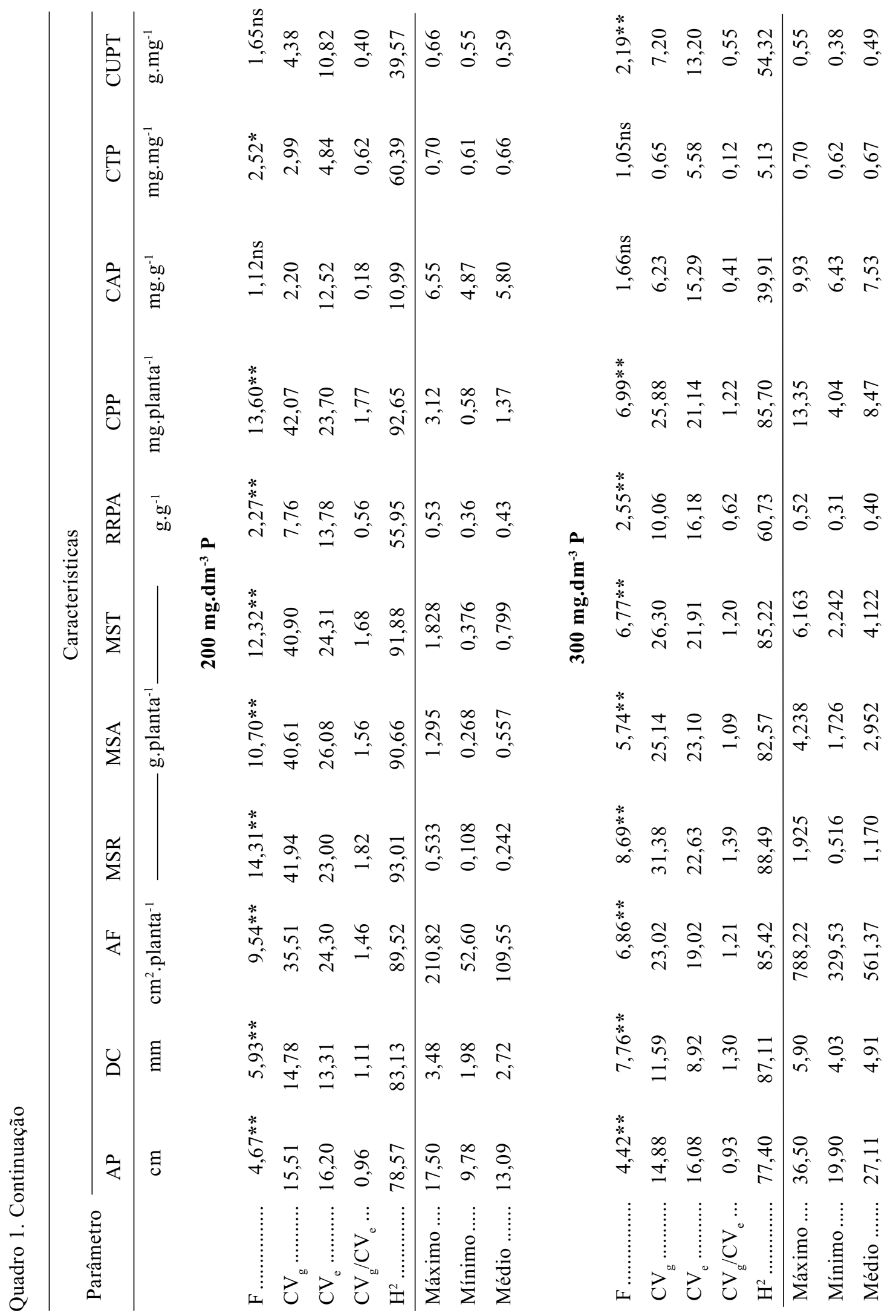


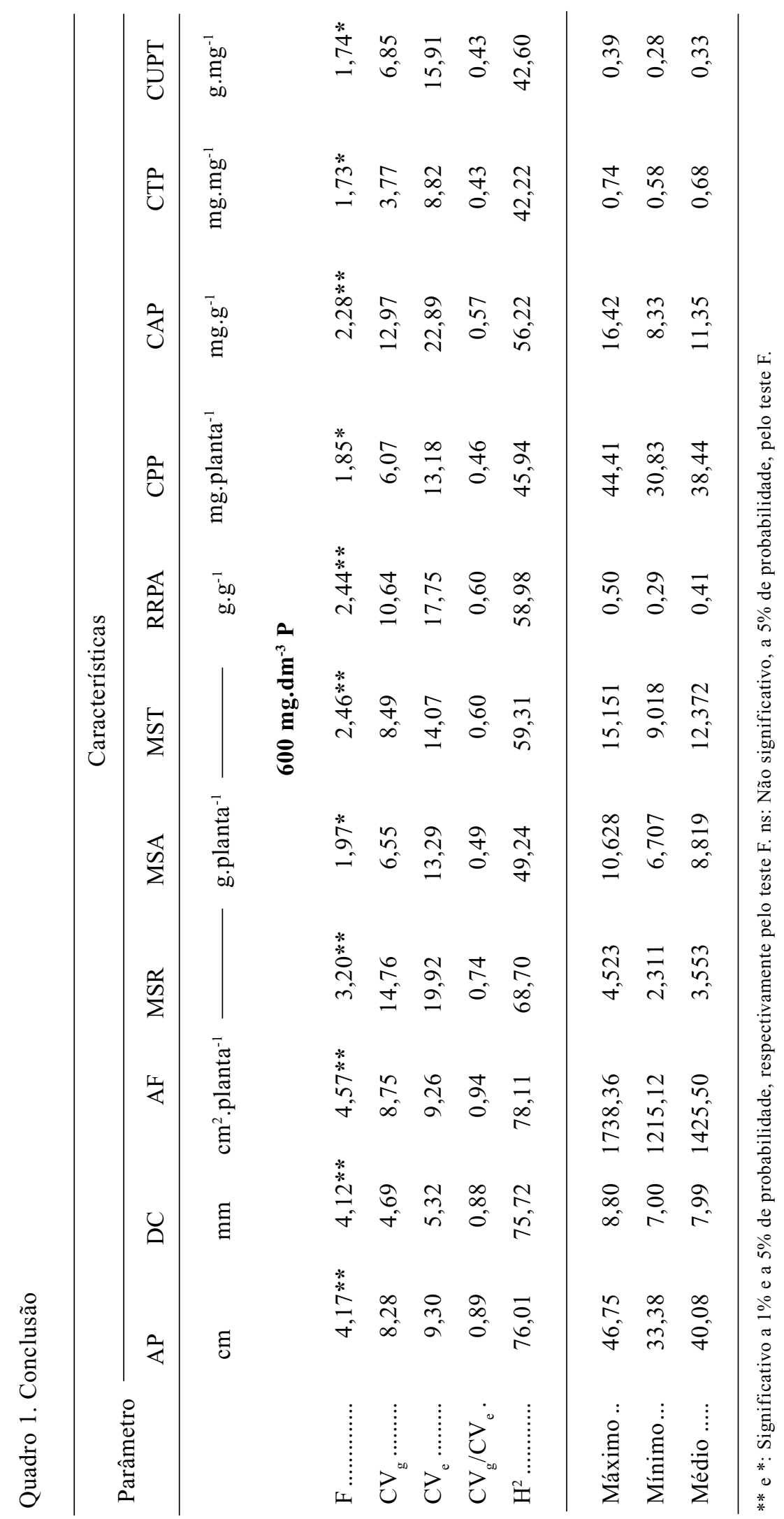


Adotando-se o critério de classificar como tolerantes e intolerantes ao baixo teor de $\mathrm{P}$, genótipos de desempenho similar na acumulação de matéria seca total em solo com alta dose de $\mathrm{P}\left(600 \mathrm{mg} \cdot \mathrm{dm}^{-3} \mathrm{P}\right)$ e com diferente capacidade produtiva em solo com baixo $\mathrm{P}\left(200 \mathrm{mg}_{\mathrm{dm}} \mathrm{dm}^{-3} \mathrm{P}\right)$, destacou-se o genótipo 21 como tolerante e os genótipos 15,10 e 6 como intolerantes, sendo a média do tolerante $471 \%$ maior do que a dos três intolerantes. Os demais exibiram um desempenho que pode, a princípio, ser considerado intermediário ou de moderada tolerância.

Quadro 2. Médias da matéria seca total (MST) de 22 genótipos de pimentão cultivados em solo com as doses de 200 (deficiente em P) e $600 \mathrm{mg} \cdot \mathrm{dm}^{-3} \mathrm{P}$ (adequado em P) e respectiva classificação dos genótipos quanto à tolerância ao baixo teor de $\mathrm{P}$

\begin{tabular}{|c|c|c|c|}
\hline \multirow{3}{*}{ Genótipos } & \multicolumn{3}{|c|}{ Doses de P } \\
\hline & \multicolumn{2}{|c|}{$200 \mathrm{mg} \cdot \mathrm{dm}^{-3}$} & \multirow{2}{*}{$\frac{600 \mathrm{mg}^{2} \mathrm{dm}^{-3}}{\mathrm{MST}}$} \\
\hline & MST & Classificação $^{(1)}$ & \\
\hline & g.planta ${ }^{-1}$ & & g.planta ${ }^{-1}$ \\
\hline 21. P-142-403-F10 .. & $1,828 \mathrm{a}$ & $\mathrm{T}$ & $12,205 \mathrm{abc}$ \\
\hline 13. P-142-215-F14 .. & $1,225 \mathrm{~b}$ & MT & $11,067 \mathrm{abc}$ \\
\hline 7. P-141-152-F13.. & $1,162 \mathrm{bc}$ & MT & $11,063 \mathrm{abc}$ \\
\hline 3. P-141-44-F13 ... & $1,123 \mathrm{bc}$ & MT & $12,788 \mathrm{abc}$ \\
\hline 8. P-141-164-F8 .... & $1,022 \mathrm{bcd}$ & MT & $12,565 \mathrm{abc}$ \\
\hline 1. P-141-4-F11 ...... & $0,978 \mathrm{~b}-\mathrm{e}$ & MT & $13,914 \mathrm{ab}$ \\
\hline 12. P-141-203-F8 .... & $0,917 \mathrm{~b}-\mathrm{e}$ & MT & $13,367 \mathrm{abc}$ \\
\hline 4. P-141-52-F8 ...... & $0,840 \mathrm{~b}-\mathrm{f}$ & MT & $14,198 \mathrm{ab}$ \\
\hline 11. P-141-195-F11 .. & $0,835 \mathrm{~b}-\mathrm{f}$ & MT & $9,018 \mathrm{c}$ \\
\hline 19. P-142-356-F10 .. & $0,831 \mathrm{~b}-\mathrm{f}$ & MT & $11,968 \mathrm{abc}$ \\
\hline 18. P-142-352-F14 .. & $0,814 \mathrm{~b}-\mathrm{f}$ & MT & $12,328 \mathrm{abc}$ \\
\hline 5. P-141-90-F14 .... & $0,718 \mathrm{~b}-\mathrm{f}$ & MT & $12,933 \mathrm{abc}$ \\
\hline 22. BGH $4531 \ldots \ldots \ldots .$. & $0,718 \mathrm{~b}-\mathrm{f}$ & MT & $11,952 \mathrm{abc}$ \\
\hline 14. P-142-232-F9 .... & $0,679 \mathrm{c}-\mathrm{f}$ & MT & $15,151 \mathrm{a}$ \\
\hline 2. P-141-27-F14 .... & $0,583 \mathrm{def}$ & MT & $12,485 \mathrm{abc}$ \\
\hline 16. P-142-319-F8 .... & $0,577 \mathrm{def}$ & MT & $11,552 \mathrm{abc}$ \\
\hline 17. P-142-320-F9 .... & $0,557 \mathrm{def}$ & MT & $12,519 \mathrm{abc}$ \\
\hline 20. P-142-378-F11 .. & $0,512 \mathrm{def}$ & MT & $11,295 \mathrm{abc}$ \\
\hline 9. P-141-177-F8 .... & 0,493 ef & MT & $10,435 \mathrm{bc}$ \\
\hline 6. P-141-150-F9 .... & $0,395 \mathrm{f}$ & I & $12,653 \mathrm{abc}$ \\
\hline 10. P-141-190-F15 .. & $0,395 \mathrm{f}$ & I & $14,209 \mathrm{ab}$ \\
\hline 15. P-142-270-F11.. & $0,376 \mathrm{f}$ & I & $12,485 \mathrm{abc}$ \\
\hline
\end{tabular}

(1) T: tolerante, MT: moderadamente tolerante, I: intolerante.

Médias seguidas de, pelo menos, uma mesma letra, não diferem estatisticamente entre si, a 5\% de probabilidade, pelo teste de Tukey. 
Diferenças significativas entre genótipos na acumulação de matéria seca total em resposta à deficiência de P também têm sido relatadas em tomate (Whiteaker et al., 1976; Coltman et al., 1985), milho (Silva \& Gabelman, 1992) e pimentão (Moura, 1996).

Não houve relação entre crescimento em solo com suprimento adequado de $\mathrm{P}$ e tolerância ao baixo teor de $\mathrm{P}$, conforme a baixa correlação encontrada entre a matéria seca total nas doses de 200 e 600 $\mathrm{mg} \cdot \mathrm{dm}^{-3} \mathrm{P}$ (coeficiente de correlação de Spearman = -0,068). Esse resultado é indicativo de que, de modo geral, os genótipos tolerantes não são responsivos, e mostra a dificuldade de desenvolver cultivares adaptados a uma ampla faixa de ambientes, e que são os de maior interesse em programas de melhoramento.

Desde que a tolerância é uma característica complexa, compreendendo vários componentes, genótipos realmente superiores são aqueles que reúnem uma série de atributos favoráveis, que lhes confere superioridade na produção biológica e/ou econômica. Assim, tolerante ao baixo teor de $\mathrm{P}$, não deve ser considerado apenas o genótipo com desempenho superior em termos de matéria seca total, mas também aquele que reúne qualidades em outras características de importância. Por exemplo, altura da planta é uma característica de grande importância em pimentão, pelas altas correlações que apresenta com produção total de frutos e peso médio de fruto (Braz, 1982); maior área foliar é importante, pois ao mesmo tempo que favorece a captação de energia solar, promove boa proteção aos frutos (Moura, 1996); da mesma forma, extenso sistema radicular é relevante para a adaptação de genótipos a solos deficientes em P, pela limitada difusão desse elemento na solução do solo (Marschner, 1986). No aspecto nutricional, genótipos eficientes na absorção, na translocação de $\mathrm{P}$ para a parte aérea e na utilização do nutriente para a conversão em biomassa, também são desejados (Horst et al., 1993). Desse modo, uma análise que leve em consideração diversos componentes importantes, simultaneamente, deve resultar em maior probabilidade de selecionar genótipos superiores, de modo que indivíduos que, pela simples análise de médias de matéria seca total, tenham sido classificados numa categoria, na medida em que outras características forem consideradas, podem ter sua classificação alterada.

Quando o objetivo é a avaliação simultânea de um conjunto de características, as técnicas de análise multivariada têm-se mostrado eficientes, por permitir combinar múltiplas informações contidas na unidade experimental, possibilitando a seleção de genótipos mais promissores com base em um complexo de variáveis (Cruz \& Regazzi, 1994). O procedimento de análise proposto por Anderson (1958) permite alocar genótipos de comportamento desconhecido em grupos conhecidos. No caso presente, o objetivo foi alocar os genótipos nos grupos tolerantes e intolerantes ao baixo teor de $\mathrm{P}$. O primeiro passo consistiu na identificação, no conjunto avaliado, dos genótipos representativos dos dois grupos de interesse. Certamente, seria pouco provável encontrar, no conjunto avaliado, genótipos que reunissem superioridade ou inferioridade em todas as características importantes. No entanto, os genótipos 21 e 6, pelo comportamento consistentemente superior e inferior, respectivamente, neste e em outros ensaios, em condições de campo e de casa de vegetação, foram considerados como padrões de tolerância e intolerância ao baixo teor de P no solo. Assim, com base nesses dois genótipos, estimaram-se as funções discriminantes dos genótipos tolerantes $\left[\mathrm{D}_{\mathrm{t}}(\mathrm{x})\right]$ e intolerantes $\left[\left(\mathrm{D}_{\mathrm{i}}(\mathrm{x})\right]\right.$ dadas por:

$\mathrm{D}_{\mathrm{t}}(\mathrm{x})=4005645,75-237771,42 \mathrm{AP}-1044322,81 \mathrm{DC}$ - 21921,21AF + 4219470,50MST - 871297,87RRPA - 5306782,50CTP;

$\mathrm{D}_{\mathrm{i}}(\mathrm{x})=4005649,75$ - 237771,61AP - 1044323,06DC 21921,22AF + 4219471,00MST - 871297,62 RRPA - 5306783,00CTP.

Verifica-se que $\mathrm{D}_{\mathrm{t}}(\mathrm{x})$ e $\mathrm{D}_{\mathrm{i}}(\mathrm{x})$ são combinações lineares das características AP, DC, AF, MST, RRPA e CTP apenas. Não se utilizaram as demais características estimadas na avaliação das funções discriminantes, ou por não terem sido estatisticamente significativas (Quadro 1), ou por problemas estatísticos, como no caso de MSR, MSA e CPP, que, sendo altamente correlacionadas com MST, proporcionam uma matriz de covariância singular, em função da 
multicolinearidade que se manifesta. Como o processo de obtenção das funções discriminantes depende da inversa da matriz de covariância fenotípica, a nãosingularidade é indispensável, o que somente foi possível com a eliminação das características MSR, MSA e CPP.

No Quadro 3 encontra-se a classificação dos genótipos quanto à tolerância ao baixo teor de $\mathrm{P}$, pela média das repetições, com base nas funções discriminantes estabelecidas. Os genótipos 21 e 6 , considerados como padrões de tolerância e intolerância respectivamente, realmente foram classificados como tal, indicando que a taxa de erro aparente, que mede o número de classificações erradas, é nula (Cruz, 1997). Conclui-se, portanto, que as funções estimadas têm consistência estatística e que as inferências a respeito dos genótipos desconhecidos são válidas.

Quadro 3. Classificação dos genótipos de pimentão quanto à tolerância ao baixo teor de $\mathrm{P}$ no solo, na dose de $200 \mathrm{mg} \cdot \mathrm{dm}^{-3} \mathrm{P}$, de acordo com as respectivas estimativas das funções discriminantes, conforme o método de Anderson (1958)

\begin{tabular}{|c|c|c|c|}
\hline Genótipos & $\mathrm{D}_{\mathrm{t}}(\mathrm{x})$ & $\mathrm{D}_{\mathrm{i}}(\mathrm{x})$ & Classificação $^{(1)}$ \\
\hline 1. P-141-4-F11 ........ & $-4466192,50$ & $-4466192,50$ & $\mathrm{I} / \mathrm{T}$ \\
\hline 2. $\mathrm{P}-141-27-\mathrm{F} 14 \ldots \ldots$ & $-4166050,50$ & $-4166050,00$ & I \\
\hline 3. P-141-44-F13 ...... & $-4743731,50$ & $-4743732,00$ & $\mathrm{~T}$ \\
\hline 4. P-141-52-F8 ........ & $-5108506,00$ & $-5108506,50$ & $\mathrm{~T}$ \\
\hline 5. P-141-90-F14 ...... & $-4667005,50$ & $-4667005,00$ & I \\
\hline 6. P-141-150-F9 ...... & $-4017259,25$ & $-4017258,50$ & I \\
\hline 7. $\mathrm{P}-141-152-\mathrm{F} 13 \ldots$ & $-5624324,00$ & $-5624325,00$ & $\mathrm{~T}$ \\
\hline 8. P-141-164-F8 ...... & $-5689081,00$ & $-5689082,50$ & $\mathrm{~T}$ \\
\hline 9. P-141-177-F8 ...... & $-4671148,00$ & $-4671147,50$ & I \\
\hline 10. $\mathrm{P}-141-190-\mathrm{F} 15 \ldots$ & $-4513826,50$ & $-4513825,50$ & I \\
\hline 11. P-141-195-F11 .... & $-5302240,00$ & $-5302241,00$ & $\mathrm{~T}$ \\
\hline 12. P-141-203-F8 ...... & $-4542468,50$ & $-4542469,00$ & $\mathrm{~T}$ \\
\hline 13. P-141-215-F14 .... & $-5446667,50$ & $-5446669,00$ & $\mathrm{~T}$ \\
\hline 14. P-142-232-F9 ...... & $-4144578,75$ & $-4144578,00$ & I \\
\hline 15. P-142-270-F11 ... & $-4005652,25$ & $-4005651,50$ & I \\
\hline 16. P-142-319-F8 ...... & $-5459071,00$ & $-5459070,50$ & I \\
\hline 17. P-142-320-F9 ...... & $-4683931,00$ & $-4683930,50$ & I \\
\hline 18. P-142-352-F14 .... & $-5253819,50$ & $-5253820,00$ & $\mathrm{~T}$ \\
\hline 19. P-142-356-F10 .... & $-5657574,50$ & $-5657575,50$ & $\mathrm{~T}$ \\
\hline 20. P-142-378-F11 .... & $-4530737,50$ & $-4530737,00$ & I \\
\hline 21. P-142-403-F10 .... & $-4005647,50$ & $-4005649,25$ & $\mathrm{~T}$ \\
\hline 22. BGH $4531 \ldots \ldots \ldots . .$. & $-5451554,50$ & $-5451554,50$ & $\mathrm{I} / \mathrm{T}$ \\
\hline
\end{tabular}

${ }^{(1)} \mathrm{T}$ : tolerante, I: intolerante, I/T: comportamento indefinido. 
Verifica-se, ainda, que foram classificados como tolerantes, além do 21 , os genótipos $3,4,7,8$, $11,12,13,18$ e 19 e, como intolerantes, além do 6, os genótipos 2, 5, 9, 10, 14, 15, 16, 17 e 20 (Quadro 3). Os genótipos 1 e 22, por exibirem estimativas de $\mathrm{D}_{\mathrm{t}}(\mathrm{x})$ e $\mathrm{D}_{\mathrm{i}}(\mathrm{x})$ de mesma magnitude, foram classificados como de comportamento indefinido. Entretanto, como o maior interesse está em genótipos consistentes em seus desempenhos nos vários ambientes, aqui representados pelas repetições, fez-se também a classificação por repetição (dados não apresentados). Assim, genótipos considerados realmente tolerantes ou intolerantes, foram aqueles cuja classificação, com base na média, foi consistente em todas as repetições, o que ocorreu com apenas uma pequena proporção dos genótipos avaliados ( $\cong 30 \%)$. Considerando apenas os genótipos de desempenho consistente, exibiram tolerância ao baixo $\mathrm{P}$, além do 21 , os genótipos 3, 7, 8 e 13 e, intolerância, além do 6 , o genótipo 15 . Com certa ressalva, pode-se ainda considerar como tolerante o genótipo 22 e, como intolerantes, 2, 5, 10 e 17. A classificação dos demais genótipos não permitiu uma conclusão segura sobre seus comportamentos.

Embora essa inconsistência na resposta possa ser atribuída a diversos fatores, a principal causa pode ter sido uma possível desuniformidade na distribuição do fertilizante fosfatado no solo, associada à colheita das plantas ainda na idade jovem. Utilizou-se como fonte de $\mathrm{P}$ o superfosfato triplo moído, que, nas doses mais baixas, correspondeu a quantidade muito pequena para cada vaso e, portanto, de difícil distribuição uniforme no solo. Uma planta em solo nessas condições teria seu crescimento retardado ou acelerado pela maior ou menor disponibilidade do $\mathrm{P}$ naquele intervalo de tempo. Esse problema é minimizado à medida que a planta explora todo o volume do vaso. Para reduzir o efeito do ambiente e de forma a tornar eficiente a seleção de plantas geneticamente superiores, tem-se dado preferência à avaliação em cultivo hidropônico ou no sistema areia-alumina (Coltman et al., 1985; Furlani et al., 1985; Silva \& Gabelman, 1992). Embora possam ser considerados meios mais artificiais, alguns resultados obtidos em solução nutritiva têm apresentado boa correspondência com os obtidos no campo (Nielsen \& Barber, 1978; Schettini et al., 1987).

\subsection{Identificação dos mecanismos responsáveis pela tolerância}

No Quadro 4 estão apresentados os dados de MSR, RRPA, das características nutricionais CAP, CTP, CUPT, dos teores de P na folha (PF), no caule (PC) e na raiz (PR) e de CPP, dos genótipos classificados como tolerantes e intolerantes à baixa disponibilidade de $\mathrm{P}$, pelos critérios univariado e multivariado. Aparentemente, um único fator foi o responsável pela diferença no crescimento observado entre os genótipos tolerantes e intolerantes. Os tolerantes, que foram 2,34 vezes mais eficientes na produção de matéria seca total, acumularam aproximadamente 2,38 vezes mais $\mathrm{P}$, em média, do que os intolerantes. Embora a biomassa das raízes secas não seja uma boa medida para descrever o sistema radicular, a maior biomassa das raízes dos genótipos tolerantes pode ser a explicação para o ocorrido. Desde que o P é transportado quase que exclusivamente via difusão para a superfície das raízes, genótipos com habilidade de desenvolver um amplo sistema radicular, tendem a ser mais tolerantes sob limitada disponibilidade de $\mathrm{P}$, principalmente por facilitar a difusão, diminuindo a distância entre o elemento e a raiz (Sattelmacher et al., 1993). Diferenças no crescimento de genótipos tolerantes e intolerantes foram também associadas à eficiência de acumulação do elemento em resposta ao tamanho do sistema radicular, tanto em milho (Furlani et al., 1985) quanto em alface (Buso \& Bliss, 1988). Não houve diferenças entre os genótipos na absorção de $\mathrm{P}$ por unidade de biomassa de raiz seca, indicando que os diversos genótipos tiveram capacidade similar em absorver o P do solo.

Crescimento preferencial das raízes em relação aos ramos, em resposta à deficiência de $\mathrm{P}$, tem sido considerado um dos mecanismos pelos quais as plantas, sob baixa disponibilidade do nutriente, obtêm mais P (Föhse et al., 1988). Embora se tenha observado um aumento na relação raiz/parte aérea, na medida 


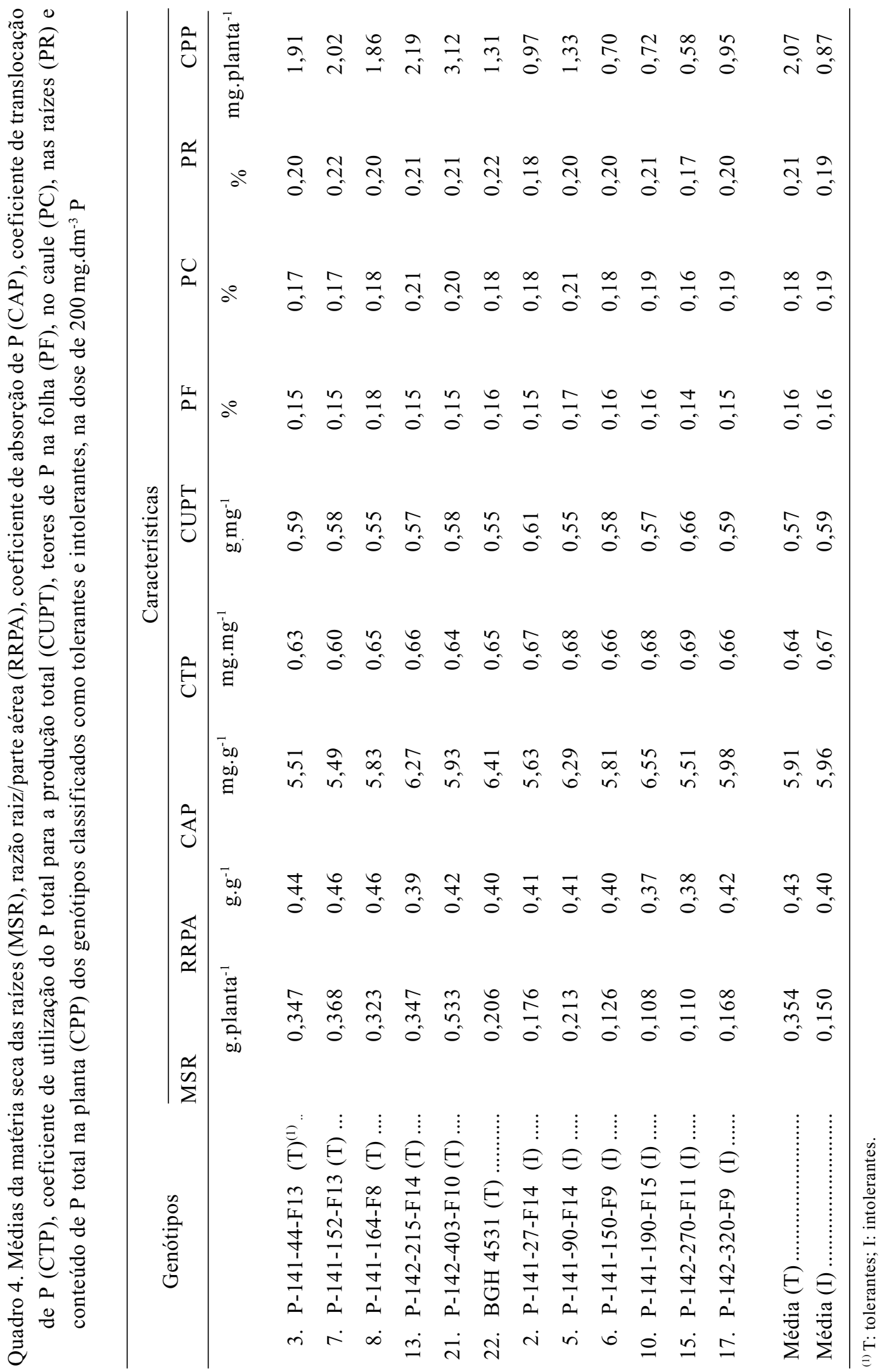


em que se diminuiu a dose de $\mathrm{P}$ aplicada no solo (Quadro 1), esse mecanismo, aparentemente, não contribuiu para diferenciar os genótipos tolerantes e intolerantes, na dose de $200 \mathrm{mg} \cdot \mathrm{dm}^{-3} \mathrm{P}$ (Quadro 4). A exemplo dos resultados aqui obtidos, maior razão raiz/parte aérea também não mostrou ser uma característica adaptativa à baixa disponibilidade de $\mathrm{P}$ no solo em tomate (Coltman et al., 1985) e alface (Buso \& Bliss, 1988). No Quadro 4, os valores de CTP indicam que a eficiência de translocação do $\mathrm{P}$ para a parte aérea também não se revelou causa de maior acumulação de $\mathrm{P}$ pelos genótipos tolerantes, conforme sugerido por Saric (1987).

Estudos têm mostrado que a atividade de fosfatases em raízes pode ser um fator bioquímico com grande contribuição para a diferenciação de genótipos sob limitada disponibilidade de P, por tornar disponível o P orgânico do solo (Helal, 1990). Entretanto, a quantificação da atividade da fosfatase ácida, realizada para o mesmo conjunto de genótipos, cultivados com as mesmas doses de $\mathrm{P}$, indicou que embora tenha havido uma redução linear na atividade da enzima com o aumento da dose de $\mathrm{P}$, não se detectaram diferenças significativas entre genótipos, quanto à atividade da enzima, na dose de $200 \mathrm{mg} \cdot \mathrm{dm}^{-3} \mathrm{P}$ (Gomide, 1996). Conclui-se, portanto, que maior atividade de fosfatases ácidas também não foi uma resposta adaptativa ao baixo teor de $\mathrm{P}$ no solo neste estudo.

Genótipos tolerantes e com outras características desejáveis podem ser usados diretamente em ensaios de produção ou em programas de melhoramento. A ampla diversidade entre eles, associada às suas qualidades agronômicas, indica que a inclusão de vários progenitores tolerantes num programa de hibridação pode ser boa estratégia. Podem ser recomendados cruzamentos envolvendo os genótipos 21 , 7, 13 e 8 . Deve-se salientar, no entanto, que o presente estudo está limitado ao crescimento vegetativo das plantas e, por isso, é recomendável avaliar esses genótipos na maturidade, em condições de campo, para verificar se a tolerância ao estresse causado por baixo teor de $\mathrm{P}$ no solo na fase vegetativa de crescimento, correlaciona-se com a tolerância e a produção de frutos no campo.

\section{CONCLUSÕES}

1. A dose de $200 \mathrm{mg} \cdot \mathrm{dm}^{-3} \mathrm{P}$ foi a mais indicada para a identificação de genótipos tolerantes à baixa disponibilidade de $\mathrm{P}$, permitindo identificar seis genótipos tolerantes, dez moderadamente tolerantes e seis intolerantes, entre os 22 avaliados.

2. A diferença no crescimento entre genótipos tolerantes e intolerantes foi devida à eficiência das plantas na absorção do $\mathrm{P}$ do solo pelas raízes.

3. Diferenças no uso interno do P não contribuíram para a diferenciação de genótipos tolerantes $\mathrm{e}$ intolerantes.

\section{REFERÊNCIAS BIBLIOGRÁFICAS}

ANDERSON, T.W. An introduction to multivariate statistical analysis. New York, John Wiley \& Sons, 1958. 345p.

BRAGA, J.M. \& DEFELIPO, B.V. Determinação espectrofotométrica de fósforo em extratos de solo e material vegetal. Revista Ceres, Viçosa, 21(113):73-85, 1974.

BRAZ, L.T. Avaliação de caracteres agronômicos e qualitativos de três cultivares de pimentão (Capsicum annuum L.) e da heterose em seus hibridos $F_{\text {. }}$ Viçosa, 1982. 75p. Tese (Mestrado em Genética e Melhoramento) - UFV, 1982.

BUSO, G.S.C. \& BLISS, F.A. Variability among lettuce cultivars grown at two levels of available phosphorus. Plant and Soil, Dordrecht, 111(1):67-73, 1988.

CHAUBEY, C.N.; SENADHIRA, D. \& GREGORIO, G.B. Genetic analysis of tolerance for phosphorus deficiency in rice (Oryza sativa L.). Theoretical and Applied Genetics, Berlin, 89(2-3):313-317, 1994.

COLTMAN, R.R.; GERLOFF, G.C. \& GABELMAN, W.H. Differential tolerance of tomato strains to maintained and deficient levels of phosphorus. Journal of American Society for Horticultural Science, Alexandria, 110(2):140144, 1985.

CRUZ, C.D. Programa Genes. Aplicativo computacional em genética e estatística. Viçosa, Editora UFV, 1997. 442p.

CRUZ, C.D. \& REGAZZI, A.J. Modelos biométricos aplicados ao melhoramento genético. Viçosa, Imprensa Universitária, 1994. 390p. 
FAGERIA, N.K. \& BALIGAR, V.C. Phosphorus-use efficiency by corn genotypes. Journal of Plant Nutrition, New York, 20(10):1267-1277, 1997.

FÖHSE, D.; CLAASSEN, N. \& JUNGK, A. Phosphorus efficiency of plants. Plant and Soil, Dordrecht, 110(1):101-109, 1988.

FURLANI, A.M.C.; BATAGLIA, O.C. \& LIMA, M. Eficiência de linhagens de milho na absorção e utilização de fósforo em solução nutritiva. Bragantia, Campinas, 44(1):129-147, 1985.

GABELMAN, W.H.; GERLOFF, G.C.; SCHETTINI, T. \& COLTMAN, R.R. Genetic variability in root systems associated with nutrient acquisition and use. HortScience, Alexandria, 21(4):971-973, 1986.

GOMIDE, M.L. Análise isozimática e atividade da fosfatase ácida em linhagens de pimentão (Capsicum annuum L.). Viçosa, 1996. 57p. Tese (Mestrado em Genética e Melhoramento) - UFV, 1996.

GOURLEY, C.J.P.; ALLAN, D.L. \& RUSSELLE, M.P. Defining phosphorus efficiency in plants. In: BARROW, N.J., ed. Plant nutrition: from genetic engineering to field practice. Netherlands, Kluwer Academic Publishers, 1993. p.363-366.

HELAL, H.M. Varietal differences in root phosphatase activity as related to the utilization of organic phosphates. Plant and Soil, Dordrecht, 123(2):161-163, 1990.

HORST, W.J.; ABDOU, M. \& WIESLER, F. Genotypic differences in phosphorus efficiency of wheat. In: BARROW, N.J., ed. Plant nutrition: from genetic engineering to field practice. Netherlands, Kluwer Academic Publishers, 1993. p.367-370.

MARSCHNER, H. Mineral nutrition of higher plants. London, Academic Press, 1986. 674p.

MOURA, W.M. Eficiência nutricional para fósforo em linhagens de pimentão (Capsicum annuum L.). Viçosa, 1996. 102p. Tese (Doutorado em Fitotecnia) - UFV, 1996.
NIELSEN, N.E. \& BARBER, S.A. Differences among genotypes of corn in the kinetics of $\mathrm{P}$ uptake. Agronomy Journal, Madison, 70(5):695-698, 1978.

OLIVEIRA, I.P.; THUNG, M.; KLUTHCOUSKI, J.; AIDAR, H. \& CARVALHO, J.R.P. de. Avaliação de cultivares de feijão quanto a eficiência no uso do fósforo. Pesquisa Agropecuária Brasileira, Brasília, 22(1):39-45, 1987.

OLIVEIRA, V.R. Diversidade genética em pimentão (Capsicum annuum L.) e controle gênico da tolerância ao baixo teor de fósforo no solo. Viçosa, 1997. 102p. Tese (Doutorado em Genética e Melhoramento) - UFV, 1997.

SARIC, M.R. Progress since the first international symposium: "Genetic aspects of plant mineral nutrition", Beograd, 1982, and perspectives of future research. Plant and Soil, Dordrecht, 99(1):197-209, 1987.

SATTELMACHER, B.; GERENDAS, J.; THOMS, K.; BLUCK, H. \& BAGDADY, N.H. Interaction between root growth and mineral nutrition. Environmental and Experimental Botany, Great Britain, 33(4):63-73, 1993.

SCHETTINI, T.M.; GABELMAN, W.H. \& GERLOFF, G.C. Incorporation of phosphorus efficiency from exotic germplasm into agriculturally adapted germplasm of common bean (Phaseolus vulgaris L.). Plant and Soil, Dordrecht, 99(1):175-184, 1987.

SILVA, A.E. da \& GABELMAN, W.H. Screening maize inbred lines for tolerance to low-P stress condition. Plant and Soil, Dordrecht, 146(1-2):181-187, 1992.

VENCOVSKY, R. Herança quantitativa. In: PATERNIANI, E. \& VIEGAS, G.P., eds. Melhoramento e produção de milho. Campinas, Fundação Cargill, 1987. v.1, p.135-214.

WHITEAKER, G.; GERLOFF, G.C.; GABELMAN, W.H. \& LINDGREN, D. Intraspecific differences in growth of beans at stress levels of phosphorus. Journal of American Society for Horticultural Science, Alexandria, 101(4):472475, 1976. 